Western Washington University

Western CEDAR

$2-1-2008$

\title{
The Tertiary Structure and Domain Organization of Coagulation Factor VIII
}

\author{
P. Clint Spiegel \\ Western Washington University, paul.spiegel@wwu.edu
}

Betty W. Shen

Chong-Hwan Chang

Jae-Wook Huh

Jeanman Kim

See next page for additional authors

Follow this and additional works at: https://cedar.wwu.edu/chemistry_facpubs

Part of the Biochemistry Commons

\section{Recommended Citation}

Spiegel, P. Clint; Shen, Betty W.; Chang, Chong-Hwan; Huh, Jae-Wook; Kim, Jeanman; Kim, Young-Ho; and Stoddard, Barry L., "The Tertiary Structure and Domain Organization of Coagulation Factor VIII" (2008). Chemistry Faculty and Staff Publications. 8.

https://cedar.wwu.edu/chemistry_facpubs/8 
Authors

P. Clint Spiegel, Betty W. Shen, Chong-Hwan Chang, Jae-Wook Huh, Jeanman Kim, Young-Ho Kim, and Barry L. Stoddard 


\title{
The tertiary structure and domain organization of coagulation factor VIII
}

\author{
Betty W. Shen, ${ }^{1}$ Paul Clint Spiegel, ${ }^{1}$ Chong-Hwan Chang, ${ }^{2}$ Jae-Wook Huh, ${ }^{2}$ Jung-Sik Lee, ${ }^{2}$ Jeanman Kim, ${ }^{2}$ Young-Ho Kim, ${ }^{3}$ \\ and Barry L. Stoddard ${ }^{1}$ \\ ${ }^{1}$ Program in Molecular Biophysics, Structure and Design, Division of Basic Sciences, Fred Hutchinson Cancer Research Center, Seattle, WA; ${ }^{2} \mathrm{Central}$ Research \\ Institute, Green Cross Corporation, Yongin, Korea; and ${ }^{3}$ Department of Life Sciences, The University of Suwon, Kyongi-do, Korea
}

Factor VIII (fVIII) is a serum protein in the coagulation cascade that nucleates the assembly of a membrane-bound protease complex on the surface of activated platelets at the site of a vascular injury. Hemophilia $A$ is caused by a variety of mutations in the factor VIII gene and typically requires replacement therapy with purified protein. We have determined the structure of a fully active, recombinant form of factor VIII (r-fVIII), which consists of a heterodimer of peptides, respectively containing the A1-A2 and $\mathrm{A} 3-\mathrm{C} 1-\mathrm{C} 2$ domains. The structure permits unambiguous modeling of the relative orientations of the $\mathbf{5}$ domains of $\mathbf{r}-\mathrm{fVIII}$. Comparison of the structures of fVIII, fV, and ceruloplasmin indicates that the location of bound metal ions and of glycosylation, both of which are critical for domain stabilization and association, overlap at some positions but have diverged at others. (Blood. 2008;111:1240-1247)

() 2008 by The American Society of Hematology

\section{Introduction}

The principal mechanism used to stop the loss of blood in mammals following vascular injury consists of a pair of overlapping proteolytic cascades called the extrinsic and intrinsic pathways. ${ }^{1-4}$ The process of blood coagulation requires extraordinary spatial and temporal regulation, which is accomplished by assembling and tethering the central proteolytic activities of these cascades at the location of transiently exposed biomolecules and cellular surfaces (Figure 1A). This includes an integral membrane protein called "tissue factor" that initiates the rapid up-regulation of the short-lived extrinsic pathway, ${ }^{5}$ and the surfaces of activated platelets, which modulate the activation of the longer-lived intrinsic pathway. ${ }^{6}$ A total of 2 homologous procoagulants, factors V and VIII (fV and fVIII), are each localized on the surface of these platelets, where they nucleate the assembly of multiprotein proteolytic complexes.

When fVIII is bound to activated platelets at the site of vascular injury, it recruits the serine protease fIXa into a complex that then catalyzes the proteolytic activation of $\mathrm{fX} .{ }^{1,4,7}$ The proteolytic activity of fIXa is enhanced by approximately 200000 -fold through its interaction with fVIII, calcium, and the phospholipid bilayer, ${ }^{8}$ corresponding to an increase of approximately $10^{9}$ in $\mathrm{k}_{\mathrm{cat}} / \mathrm{K}_{\mathrm{M}}$.

The full-length, unprocessed fVIII protein consists of 2332 amino acid residues and has the domain structure A1-A2-B-A3-C1$\mathrm{C}^{9-12}$ (Figure 1B). The $3 \mathrm{~A}$ domains are each approximately 330 residues, and approximately $40 \%$ identical to each other and to the copper-binding protein ceruloplasmin. ${ }^{13}$ The $\mathrm{C}$ domains are smaller (approximately 160 residues) and are more distantly related to various members of the discoidin protein fold family, such as galactose oxidase. ${ }^{14-17}$ The $\mathrm{B}$ domain has no known structural homologs, is heavily glycosylated, and is relatively dispensible for procoagulant activity. fVIII is initially processed by proteolytic cleavage events that remove a large portion of the B domain, generating a heterodimer that circulates in a tight complex with von Willebrand factor (VWF). ${ }^{18}$ This interaction is essential for maintaining stable levels of fVIII in circulation. ${ }^{19}$ Upon vascular injury, further proteolytic processing generates activated factor VIIIa (fVIIIa), a heterotrimer (A1/A2/A3-C1-C2) that is released from VWF and binds to activated platelets. ${ }^{18}$

The carboxy-terminal 159 amino acids of fVIII comprise its C2 domain, which is involved in binding to VWF and primarily responsible for binding to platelet membrane surfaces. This latter binding interaction is dependent on the transient, specific exposure of phosphatidylserine (PS) head groups on the outer leaflet of activated platelet membranes. ${ }^{20-23}$ The VWF and membranebinding activities of the $\mathrm{C} 2$ domain appear to be competitive and mutually exclusive..$^{23-26}$

Several structural models are available to the coagulation community for analysis of fVIII. A hypothetical model of the fVIII "A" domains has been generated from the crystal structure of ceruloplasmin. ${ }^{13}$ The structures of the $\mathrm{C} 2$ domain of $\mathrm{fV}$ and $\mathrm{fVIII}$ have been solved to high resolution, ${ }^{16,27,28}$ and a homology model of the fVIII $\mathrm{C} 1$ domain has been described. ${ }^{29}$ Complementing those studies is a model of the full-length fVIII heterodimer, generated from 2D electron diffraction studies, which provides a low-resolution (approximately $15 \AA$ ) view of the overall disposition and orientations of the individual fVIII domains. ${ }^{30}$ Finally, a $2.8-\AA ̊$ resolution structure of a portion of $\mathrm{fV}$ (missing its A2 domain) has also been reported..$^{31}$

\section{Methods}

A recombinant form of fVIII (r-fVIII) was expressed, secreted, and purified from Chinese hamster ovary $(\mathrm{CHO})$ cells as a heterodimer in the presence of VWF as previously described. ${ }^{32}$ The heterodimeric species of r-fVIII
Submitted August 29, 2007; accepted October 24, 2007. Prepublished online as Blood First Edition paper, November 1, 2007; DOI 10.1182/blood-2007-08109918.

B.W.S. and P.C.S. contributed equally to this study.
The online version of this article contains a data supplement.

The publication costs of this article were defrayed in part by page charge payment. Therefore, and solely to indicate this fact, this article is hereby marked "advertisement" in accordance with 18 USC section 1734.

(C) 2008 by The American Society of Hematology 
A

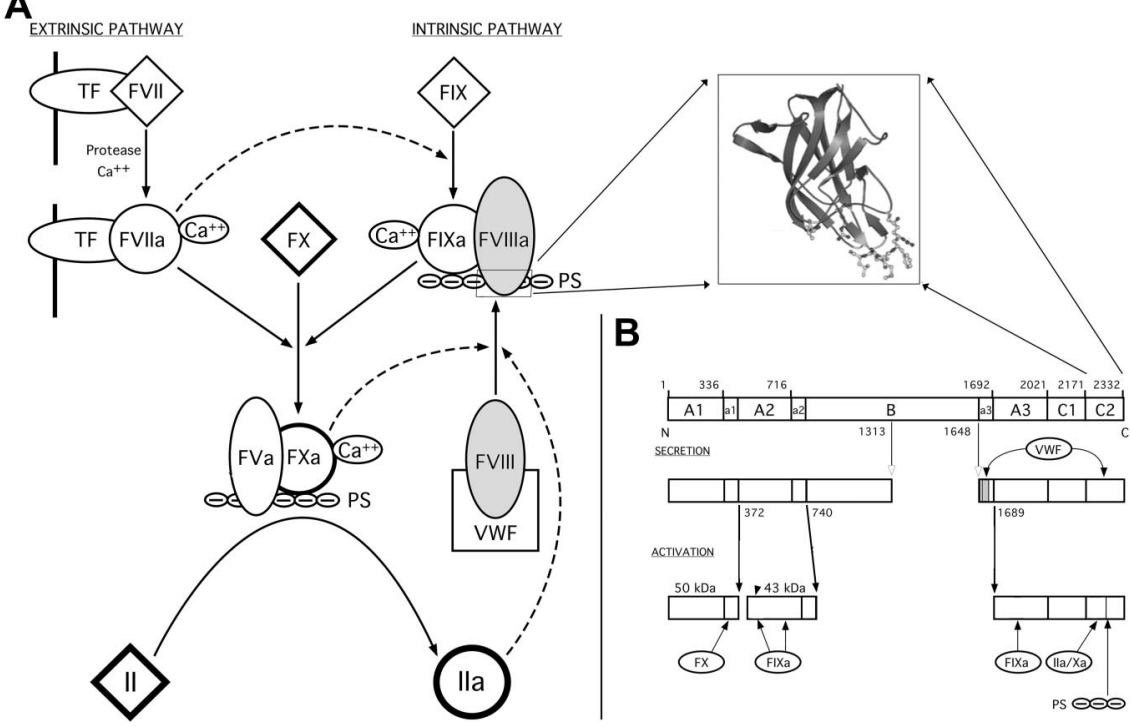

Figure 1. Factor VIII and the coagulation cascade. (A) The blood coagulation cascade consists of 2 pathways (extrinsic and intrinsic) that are initiated by the exposure of tissue factor (TF) or phosphatidylserine groups (PS) of activated platelet membranes to circulating protein factors, respectively. fVIII is a plasma glycoprotein that acts as an initiator and regulator of the intrinsic pathway. Upon proteolytic activation by either $\mathrm{fXa}$ or thrombin, fVIIla dissociates from VWF, associates with the flXa serine protease, and directs the localization of the resulting complex to the membrane surface of activated platelets via an interaction with its C-terminal $\mathrm{C} 2$ domain (structure in inset). The membrane-bound complex between fVIIla and flXa complex functions to proteolytically activate fX, which then activates thrombin (fII). (B) Domain structure of fVIII. fVIII is synthesized as a single polypeptide chain of 2332 residues. Based on sequence homology, fVIII has the domain structure A1-A2-B-A3-C1-C2. Linker regions between domains are denoted with lowercase letters ("a1," etc). The location of domain boundaries and primary sites of proteolytic processing during secretion and activation are denoted by residue numbers. The circulating fVIII heterodimer is associated with VWF primarily through interactions with the "a3" acidic region at the light chain N-terminus and with the C2 domain. Various proteases interact with the activated heterotrimer at positions denoted at the bottom panel. Membrane association is primarily accomplished through the $\mathrm{C} 2$ domain; its deletion completely abrogates binding of fVIII to platelet surfaces.

consists of a heavy chain (A1 and A2 domains) and a light chain (residues 1563-1648 of the B domain and the entire A3, C1, and C2 domains). Purified r-fVIII was pooled, concentrated to $0.5 \mathrm{mg} / \mathrm{mL}$, and stored at $-70^{\circ} \mathrm{C}$ in storage buffer ( $50 \mathrm{mM}$ imidazole [pH 6.7]; $410 \mathrm{mM} \mathrm{NaCl}, 4 \mathrm{mM}$ $\mathrm{CaCl}_{2}, 0.1 \% \mathrm{wt} / \mathrm{vol} \mathrm{PEG} 1000$, and $0.001 \% \mathrm{wt} / \mathrm{vol}$ Tween 80 ). This truncated variant of fVIII maintains procoagulant activity and can be subsequently activated by thrombin.

For crystallization purposes, r-fVIII was concentrated to $2 \mathrm{mg} / \mathrm{mL}$ by vacuum dialysis at $4^{\circ} \mathrm{C}$ against the storage buffer listed. r-fVIII was subsequently crystallized by hanging drop reverse-vapor diffusion at $4^{\circ} \mathrm{C}$ against reservoirs containing $8 \%$ to $12 \%$ (wt/vol) PEG8000, 100 to $300 \mathrm{mM} \mathrm{NaCl}$, and $50 \mathrm{mM}$ Tris- $\mathrm{HCl}$ (pH 6.5-7.5). Reverse-vapor diffusion occurs due to the higher salt concentration residing in the r-fVIII storage buffer, leading to a reduction in salt concentration in the protein drop that causes crystallization. Prior to data collection, crystals were cryoprotected with either 30\% (vol/vol) DMSO or 25\% to $30 \%$ glycerol and flash-frozen in liquid nitrogen. Data collection was performed at Beamline 5.0.2 at the Advanced Light Source (Berkeley, CA). Data were indexed, refined, and integrated with D*TREK (Molecular Structure Corporation, The Woodlands, TX) ${ }^{33}$ and scaled using the program SCALA (CCP4, Daresbury Laboratory, United Kingdom). ${ }^{34}$ The space group was determined to be $\mathrm{P} 4_{1} 2{ }_{1}$ by analyses of symmetry and systematic absences, and by examination of unbiased anomalous difference, isomorphous difference and omit maps. Particularly obvious validating features of these maps, indicating the position of bound metals and oligosaccharide structures, are shown in Figure 2.

The initial crystals used for molecular replacement were cross-linked (by transient 5 -minute exposure to $0.05 \% \mathrm{vol} / \mathrm{vol}$ glutaraldehyde, followed by cryocooling) and diffracted to approximately $4.5-\AA$ resolution. Subsequent crystals were larger, more robust, and diffracted up to approximately $3.7 \AA$ without requiring cross-linking. One of these specimens was used for final rebuilding and refinement (Tables 1,2 ). The unit cell parameters for the crystal used for the final refinement are $\mathrm{a}=\mathrm{b}=134.8 \AA$ and $\mathrm{c}=358.4 \AA$.

The phasing of the r-fVIII crystal structure was performed iteratively by molecular replacement, using the programs Phaser (CCP4) and EPMR. ${ }^{35}$ First, the 3 A domains of fVIII were simultaneously located and placed with the program Phaser using a search model consisting of a polyalanine peptide chain containing those domains, which were derived from the crystal structure of ceruloplasmin..$^{13}$ The correct solution to this search was well above background, with the top $2 \mathrm{Z}$-scores for the rotation search corresponding to 7.6 and 3.1, respectively; the top $2 \mathrm{Z}$-scores for the translation search were 23.7 and 13.5. A similar search using the coordinates of the A1 and A3 domains from the structure of inactive "A2-deleted" $\mathrm{fV}^{31}$ was unsuccessful. The position of each of the A domains was further validated by removing each domain from the solution and performing a subsequent search to find each domain independently. The position of the $\mathrm{C} 1$ domain was then determined, using a model of $\mathrm{C}^{29}$ derived from the structure of the fVIII C 2 domain $^{16}$ as a search template. Last, the position of the $\mathrm{C} 2$ domain was determined using the program EPMR with the previous solutions combined and used as a static structure while the fVIII C2 domain crystal structure was used as a search model. The resulting solution for the fVIII C2 domain generated a correlation coefficient of 0.601 and an R-factor of 50.8 .

The relative orientations of the fVIII domains resembles the orientations of the same domains that were independently determined in the crystal structure of a fragment of $\mathrm{fV}^{31}$ That model was not used at any stage of phase determination in this study. Additional protein models that did not result in an obvious molecular replacement solution were all-atom ceruloplasmin $^{36}$ and a model derived from electron diffraction data for fVIII. ${ }^{30} \mathrm{In}$ the latter structure, the orientation of the $2 \mathrm{C}$ domains differ significantly from those found in the crystal structure reported here.

Homology models of the $3 \mathrm{~A}$ domains and the $\mathrm{C} 1$ domain were constructed using the ROBETTA protein structure prediction server. ${ }^{37}$ The resulting models were superimposed on the molecular replacement solution and used as starting models for rigid body refinement using the CNS program, ${ }^{38}$ initially for the entire structure as one rigid group, and subsequently parsed into individual domains as individual rigid groups. After initial simulated annealing protocols, the values of $R_{w o r k}$ and $R_{\text {free }}$ were 0.398 and 0.459 , respectively, at which point model rebuilding was commenced against unbiased composite omit maps. During the iterative process of rebuilding and refinement, sequentially higher quality data sets were generated as shown in Tables 1,2, and were used accordingly.

Model building was done with the program COOT $^{39}$ against SIGMAAweighted Fourier and difference maps, and the structure was refined using 
A

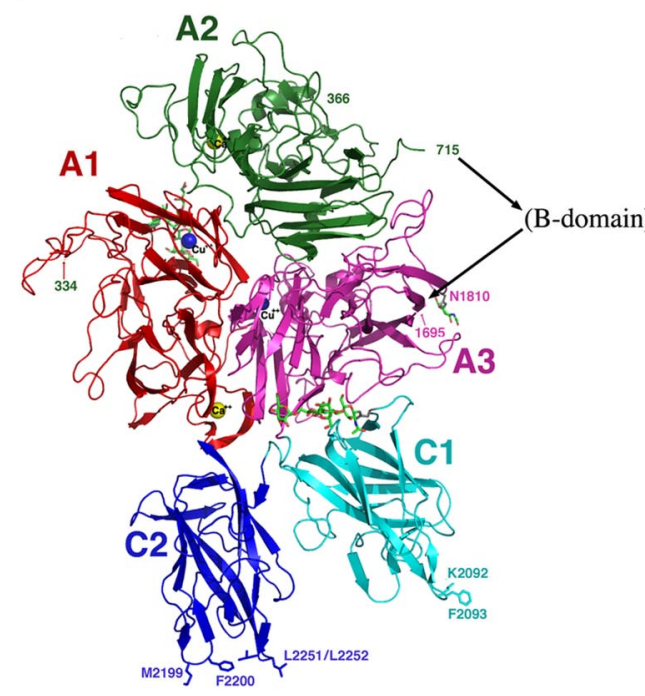

C
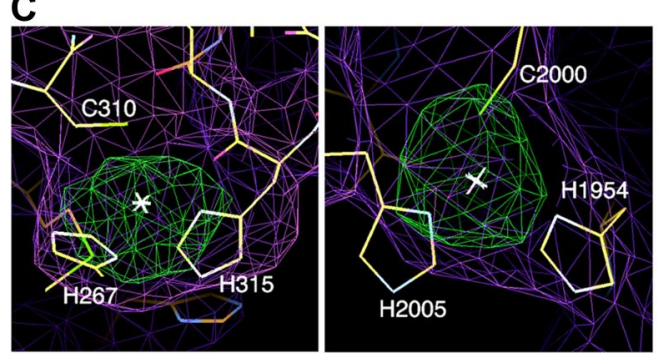

B

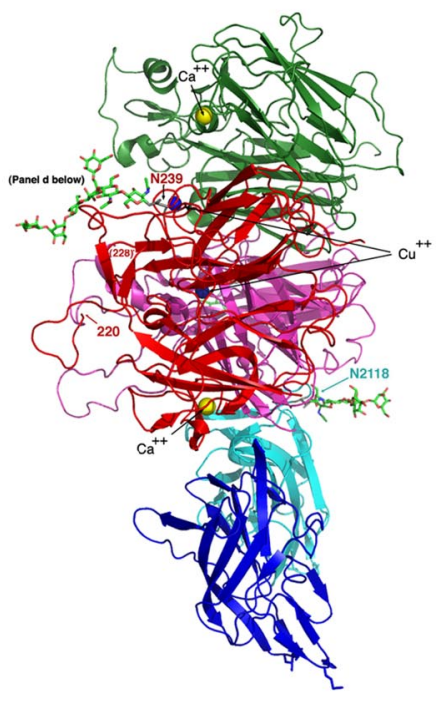

D

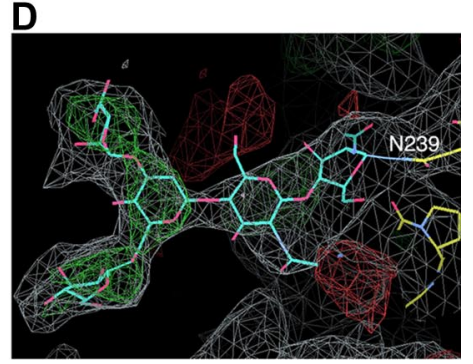

Figure 2. The structure of the B domain deleted fVIII heterodimer. $(A, B)$ The fVIII domains are individually labeled and colored. The C-terminal end of the heavy chain and the $\mathrm{N}$-terminal end of the light chain are indicated with residue numbers (715 and 1695). Residues 1563 to 1694 (the $\mathrm{N}$-terminal 80 residues of the light chain) are present in the construct but are poorly ordered. The $\mathrm{N}$ - and $\mathrm{C}$-termini of 2 additional disordered regions are also indicated with residue numbers: residues 220 and 228 that flank a surface loop in the A1 domain (right panel) and residues 334 and 366 that flank the linker region between the A1 and A2 domains (left panel). Shown are 2 bound calcium ions, 2 bound copper ions, and 3 well-ordered and visible $\mathrm{N}$-linked oligosaccharide structures. Shown and labeled for reference are 4 residues on the $\mathrm{C} 2$ domain that are thought to be involved in membrane binding and a similarly positioned pair of residues on the $\mathrm{C} 1$ domain (C) Anomalous difference peaks at the sites of bound copper ions buried in the $\mathrm{A} 1$ and $\mathrm{A} 3$ domains (contoured at $5 \sigma$; both are approximately $9 \sigma$ peak height overall). The residues involved in metal binding at this site are conserved in the analogous copper-binding site in ceruloplasmin, but are diverged from fV. (D) Difference density for 1 of $3 \mathrm{~N}$-linked oligosaccharide structures, which modifies Asn239 in the A1 domain. The density is readily apparent for the entire pentameric polysacharide mannose core of an $\mathrm{N}$-linked sugar, and represents unbiased density prior to any modeling of the covalent modification.
CNS after randomly removing $5 \%$ of the measurements to monitor the free $\mathrm{R}$-factor $\left(\mathrm{R}_{\text {free}}\right)$. Due to the low resolution of the $\mathrm{X}$-ray data in this study, all modifications of the structure during model building and refinement were evaluated and accepted based on their effect on the value of the $R_{\text {free }}$ and on the difference between $R_{\text {free }}$ and $R_{\text {work }}$, rather than solely on the value $R_{\text {work }}$ (which is more subject to model bias). During the refinement, features of unbiased electron density that were correlated with previously unmodeled structural features (such as the obvious presence of an N-linked, bifurcated mannose core glycosyl modification of Asn1810, and strong features of anomalous density for bound metal ions, as shown in Figure 2) provided clear validation of the molecular replacement solution and subsequent refinement. The final model corresponds to $\mathrm{R}_{\text {work }} / \mathrm{R}_{\text {free }}$ values of $0.289 / 0.341$ and geometric root mean square deviation (RMSD) values of $0.0092 \AA$ (bond distance) and $1.69^{\circ}$ (bond angles). Data and refinement statistics are provided in Tables 1 and 2. A comparison of the refinement statistic with structures at similar resolution in the Protein Databank (PDB) database and the Ramachandran plot of the current structure are provided in Figures $\mathrm{S} 1, \mathrm{~S} 2$ (available on the Blood website; see the Supplemental Materials link at the top of the online article).

\section{Results}

\section{Structure and domain organization}

We crystallized a fully active form of engineered r-fVIII, which consists of a heterodimer of the A1-A2 domains (the "heavy chain") and the A3-C1-C2 domains (the "light chain"). The structure permits unambiguous modeling of the relative orientations of all 5 domains of r-fVIII as well as identification of sites of glycosylation and metal binding (Figure 2). While the $\mathrm{C} 2$ domain is loosely tethered to the structure and appears capable of significant motion, the $\mathrm{C} 1$ domain forms an extensive hydrophobic interface with the A3 domain and is likely locked into a single docked position and conformation.

The structures of the individual domains within fVIII are quite similar to available crystal structures of homologous proteins. The 3 A domains can be superimposed on those from ceruloplasmin ${ }^{13}$

Table 1. Data collection and processing statistics

\begin{tabular}{|c|c|c|c|c|}
\hline Crystal & F8_w (cross-linked) & Nat-7 & Nat-4b & Nat-4c \\
\hline Wavelength, $\AA$ & 1.0246 & 1.00 & 1.00 & 1.00 \\
\hline Unit cell, $\AA$ & $a=b=134.7, c=353.6$ & $a=b=134.5, c=359.7$ & $a=b=134.6, c=359.5$ & $a=b=134.8, c=358.4$ \\
\hline Resolution, $\AA$ & $30-4.5(4.6-4.5)$ & $100-4.3(4.4-4.3)$ & $100-3.9(4.0-3.9)$ & $100-3.7(3.76-3.70)$ \\
\hline No. reflections (no. unique reflections) & 308594 (20 241) & $133617(42517)$ & $199559(28509)$ & $189184(34208)$ \\
\hline Redundancy & 15.2 & 3.1 & $7.0(2.9)$ & $5.5(3.0)$ \\
\hline $\mathrm{I} / \sigma(\mathrm{I})^{*}$ & $19.5(5.4)$ & $17.7(3.5)$ & $12.9(1.0)$ & $13.7(0.91)$ \\
\hline Completeness, \%* & $100(100)$ & $97.7(98.6)$ & $92.5(61.3)$ & $94.5(67.3)$ \\
\hline $\mathrm{R}_{\text {sym }}{ }^{*}$ & $0.161(0.682)$ & $0.084(0.553)$ & $0.092(0.735)$ & $0.096(0.798)$ \\
\hline
\end{tabular}

Space group is $\mathrm{P} 4{ }_{1} 2{ }_{1} 2$.

${ }^{\star}$ Numbers in parentheses are statistics from the highest-resolution shells. 
Table 2. Model refinement statistics

\begin{tabular}{ll}
\hline Resolution, $\AA$ & $100-3.7(3.76-3.7)$ \\
$\mathrm{R}_{\text {work }}$ & $0.289(0.470)$ \\
$\mathrm{R}_{\text {free }}$ & $0.341(0.485)$ \\
$\mathrm{RMSD}$ bond, $\AA$ & 0.0092 \\
$\mathrm{RMSD}^{\circ}$ angle, & \\
\hline Protein residues/average B-factor, no. & 1.69 \\
Carbohydrate moieties/average B-factor, no. $^{\circ}$ & $1315 / 160.1$ \\
Metal ions/average B-factor, no. & $13 / 192.0$ \\
\hline Ramachandran distribution, no. residues (\%) & $4 / 158.5$ \\
\hline
\end{tabular}

with an overall RMSD of $1.7 \AA$, and can be similarly superimposed on the $\mathrm{A} 1$ and $\mathrm{A} 3$ domains from $\mathrm{fV}$ with an RMSD of $2.2 \AA$. The $\mathrm{C} 2$ domain is closely related to the previously determined crystal structure of the isolated domain ${ }^{16}$ (RMSD, $1.5 \AA$ ). Comparison of the 2 individual $\mathrm{C}$ domains with those from the crystal structure of fV gives RMSD values of approximiately $1.9 \AA$.

Across the structure of the fVIII heterodimer, 4 regions are disordered (Figure 2): 2 short surface loops within the A1 domain (residues 34-38 and 213-227), the linker region between the A1 and A2 domains (residues 335-366), and a long 155-residue region spanning the end of the A2 domain, through the truncated portion of the $\mathrm{B}$ domain, and into the $\mathrm{N}$-terminal region of the $\mathrm{A} 3$ domain. The remaining portion of the light chain (A3-C1-C2) is well ordered (except for its final 3 residues). The $\mathrm{N}$-terminal residues of the light chain are critical for binding to VWF and are proteolytically truncated by thrombin during r-fVIII activation; it is therefore likely that they are only structurally ordered within the circulating r-fVIII/vWF complex.

The orientation of the $\mathrm{C} 1$ domain is similar to that observed in the crystal structure of $\mathrm{fV}^{31}$ (which is missing its $\mathrm{A} 2$ domain), but is rotated by approximately $90^{\circ}$ relative to the model of r-fVIII generated from previous $2 \mathrm{D}$ electron diffraction studies ${ }^{30}$ (Figure 3). The $\mathrm{C} 1$ domain is tightly associated with the A3 domain of the light chain, creating a $1200-\AA^{2}$ aromatic/ hydrophobic interface that buries approximately 20 residues and a well-ordered N-linked high-mannose glycosyl modification of N2118 (Figure 4A). This interface includes several aromatic residues (Y1748, Y2017, Y2105, and W2112), 3 prolines (P1865, P2142 and P2143), several aliphatic residues (L1747, L1752, V1933, L2015 and I2145), and a large number of hydrophilic residues, effectively locking the $\mathrm{C} 1$ domain into place within the fVIII light chain. Of the residues in the A3-C1 interface, at least 6 (L1752, N2015, and Y2017 and 2105, $\mathrm{R} 2116$, and T2122) are sites of missense mutations associated with hemophilia A.

In contrast, the $\mathrm{C} 2$ domain is relatively loosely tethered to the fVIII molecule (Figure 4A), displaying a small $400-\AA^{2}$ interface to the $\mathrm{C} 1$ domain and a $200-\AA^{2}$ interface with the A1 domain, both of which are comprised primarily of hydrophilic residues. However, the observed orientation of the $\mathrm{C} 2$ domain is similar to that observed in the fV crystal structure, indicating that this domain orientation is a reproducible feature of the full-length heterodimer (Figure 3). The respective interactions of the $\mathrm{C} 1$ and $\mathrm{C} 2$ domains within the fVIII molecule agree with their observed behavior in solution when expressed as isolated domains: the $\mathrm{C} 1$ domain is insoluble and cannot readily be purified, while the isolated $\mathrm{C} 2$ domain is well behaved in solution at high concentrations and displays specific binding to both plasma membranes and to recombinant VWF constructs (behaviors that mimic its role in the full-length coagulation factor). ${ }^{40,41}$
Figure 3. Comparisons of $\mathrm{fV}$ and fVIII structures. The relative domain orientations of $\mathrm{r}-\mathrm{fVIII}$ with a previously reported model generated from electron diffraction studies, and with $2.8-\AA$ resoloution crystal structures of ceruloplasmin and of an inactive (A2-deleted) $\mathrm{fV}$ construct are shown. The structures are all oriented similarly with respect to the A domains. Metals ions modeled in the various structures as calcium and copper are indicated as yellow and blue spheres, respectively. The docked orientations of the $\mathrm{C}$ domains of $\mathrm{r}$-fVIII, and their interactions with each other and with the $\mathrm{A} 3$ domain of the light chain, are both rotated by approximately $90^{\circ}$ with respect to the electron diffraction model, but in excellent agreement with their homologous domains in $\mathrm{fV}$. The box shows superposition of full-length fVIII (colored by domains) versus inactive (A2-deleted) fV (shown in orange). The RMSD for aligned $\alpha$-carbons is approximately $2 \AA$.
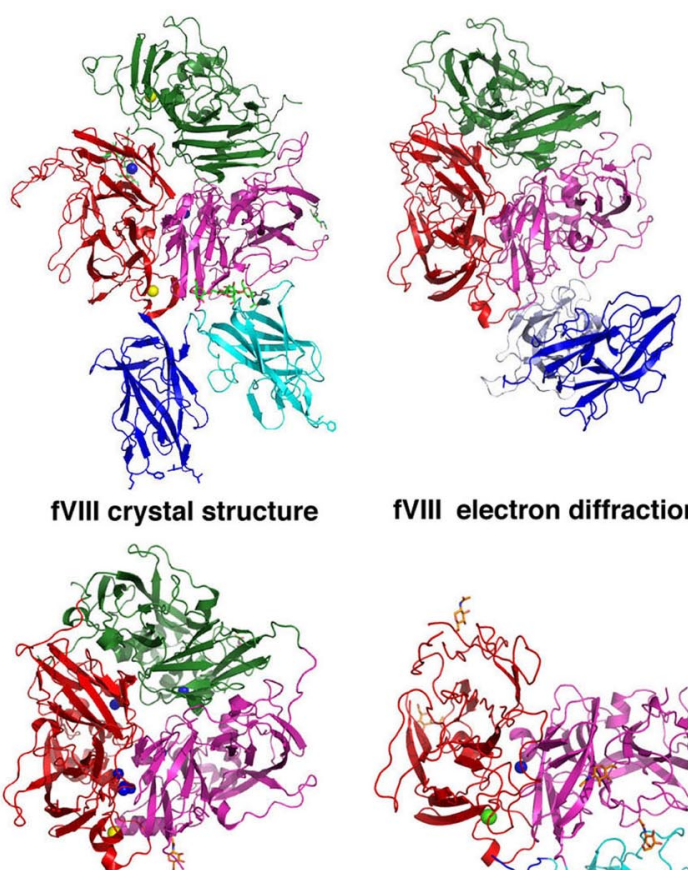

fVIII electron diffraction
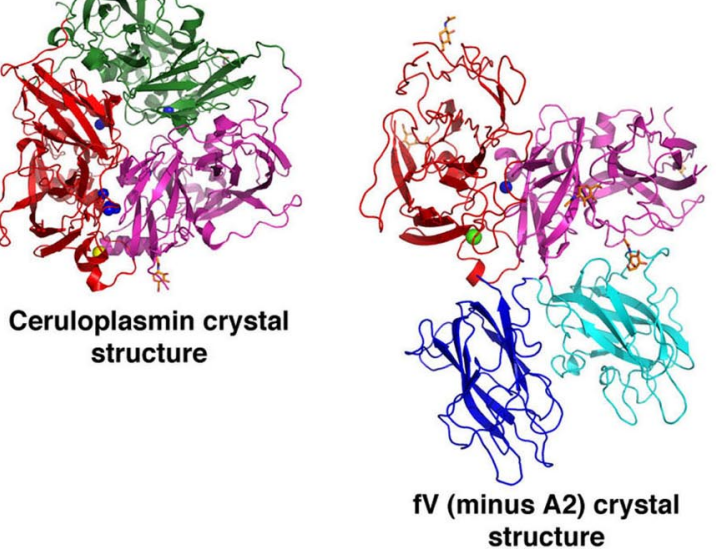

fVIII and fV crystal structures superimposed $A$ domains 
A

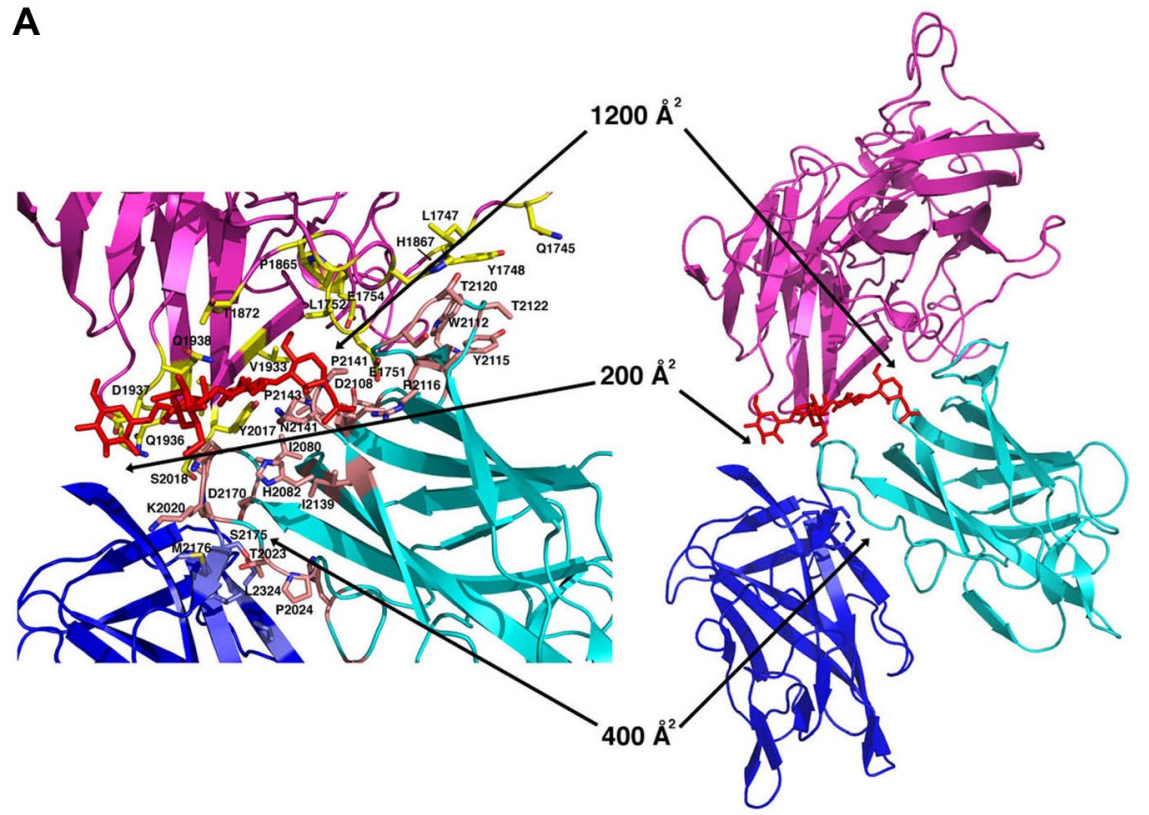

Figure 4. Interface dimensions and packing in the r-fVIII molecule. (A) The light chain and packing of the $\mathrm{C}$ domains. The $\mathrm{C} 1$ domain (cyan ribbon and pink side chains) is engaged in an extensive docked interaction against the $\mathrm{A} 3$ domain (light magenta ribbon and yellow side chains) that involves multiple aromatic, aliphatic, and hydrophilic side chains, several of which are sites of missense mutations associated with protein dysfunction and hemophilia A. An N-linked glycosyl modification (red) is also involved in the C1-A3 interface. In contrast, the $\mathrm{C} 2$ domain (blue ribbon and side chains) is loosely tethered to the r-fVIII molecule, displaying small, entirely hydrophilic interfaces with the $\mathrm{A} 3$ and $\mathrm{C} 1$ domains. Interestingly, a number of residues in these interfaces are also associated with hemophilia. (B) Packing of the trimer of A domains and close up of the packing of the A2 domain (green) against the A3 domain, with residues that have been previously mutated to create engineered disulfide cross-links indicated. These residues are located on 2 adjacent loops in the A domains, which appear to display sufficient flexibility to permit covalent disulfide formation while maintaining r-fVIII activity in vivo.

B

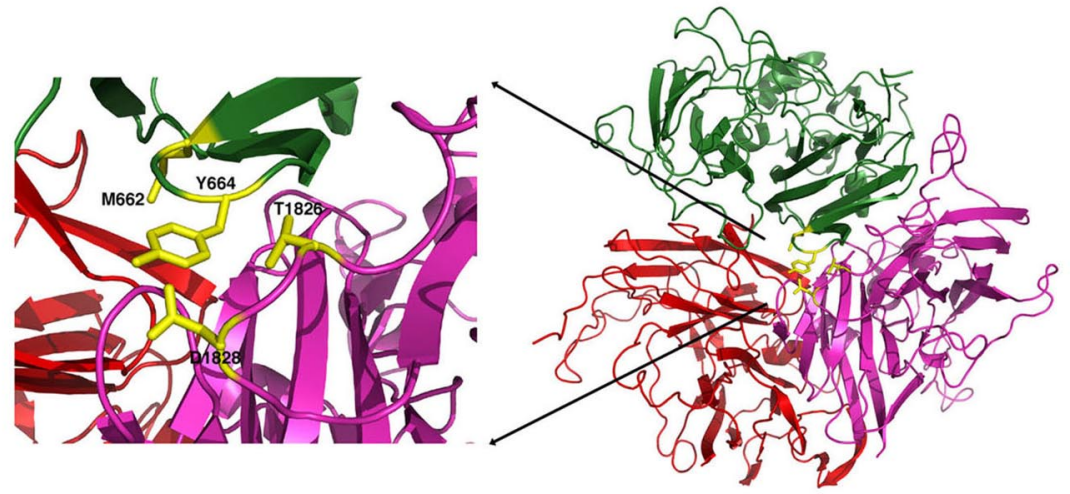

\section{Bound metal ions and sites of glycosylation}

In addition to the protein domains, 4 bound metal ions and 3 sites of glycosylation are observed in the structure (Figure 2). Acidic residues from individual A domains ligate two of the metals, which are modeled as calcium ions. The first calcium, found in the A1 domain, is tightly coordinated by a single glutamate (E110) and 3 aspartate residues (D116, D125, and D126), and is located at a position within the protein fold that is also occupied by calcium in both ceruloplasmin ${ }^{36}$ and in $\mathrm{fV}^{31}$ The second modeled calcium is coordinated by 2 aspartate residues (D538 and D542) in the A2 domain.

The 2 additional metal ions, one each in the $\mathrm{A} 1$ and $\mathrm{A} 3$ domains, are observed in anomalous difference Fourier maps (Figure 2C) and are modeled as copper ions. Previous biochemical studies have indicated the presence of one or more bound copper ions in these domains in both human $\mathrm{fVIII}^{42}$ and in $\mathrm{fV}^{43}$ and a functional role for bound copper in association of the A1 and A3 domains. ${ }^{44}$ This property reflects the relationship of both of these coagulation factors to the copper-binding protein ceruloplasmin, which contains multiple copper ions bound near the A1-A3 domain interface. Comparison of these domains among all 3 proteins indicates that their copper-binding functions are maintained, although with significant divergence in the identity and position of the side chains that coordinate the metal ion (Figure S3).
Density for the first bound copper in fVIII is observed in the A3 domain (Figures 2C,S3), coordinated by 2 histidine residues (H1954 and H2005) and a single cysteine (C2000). This metal binding site is conserved in ceruloplasmin, which is occupied by a similarly located copper ion. ${ }^{36} \mathrm{In} \mathrm{fV}$, a single bound copper ion is also located near the A1-A3 boundary, again coordinated entirely by residues from the A3 domain (H1802, H1804, and D1844). These amino acid ligands differ from fVIII, but are also partially conserved with ceruloplasmin.

A second bound copper ion is buried in the A1 domain of fVIII, and is also coordinated by 2 histidine residues (H267 and H315) and a single cysteine (C310; Figure 2C). The metalbinding residues in this site are again conserved in ceruloplasmin, but not in $\mathrm{fV}$ (where the corresponding residues are F239, $\mathrm{H} 207$, and S282).

Overall, the metal-binding function of fVIII appears to be more closely related to ceruloplasmin than to $\mathrm{fV}$. $\mathrm{fV}$ and $\mathrm{fVIII}$ each share homology in copper-binding residues with ceruloplasmin, but not with each other; this may indicate that the 2 coagulation factors have independently diverged from a common copper-binding ancestor. It is possible that additional or alternate copper binding sites may be occupied in various fVIII and/or fV constructs, depending on the precise nature of the expression system and cell line. The encorporation of copper ions in proteins, unlike transition metals such as calcium and magnesium, is known to often require 
Figure 5. Location of hemophilia A-associated missense mutations across the r-fVIII structure. (A) The disease phenotype/symptom designations of "mild," "moderate," and "severe" are taken from the HamSters database ${ }^{48}$ and correspond to plasma fVIIIa activity levels of $6 \%$ to $30 \%, 1 \%$ to $5 \%$ and less than $1 \%$, respectively. (B) The presence of mutations in the interfaces of the $\mathrm{C}$ domains with each other and with the $A 3$ domain.
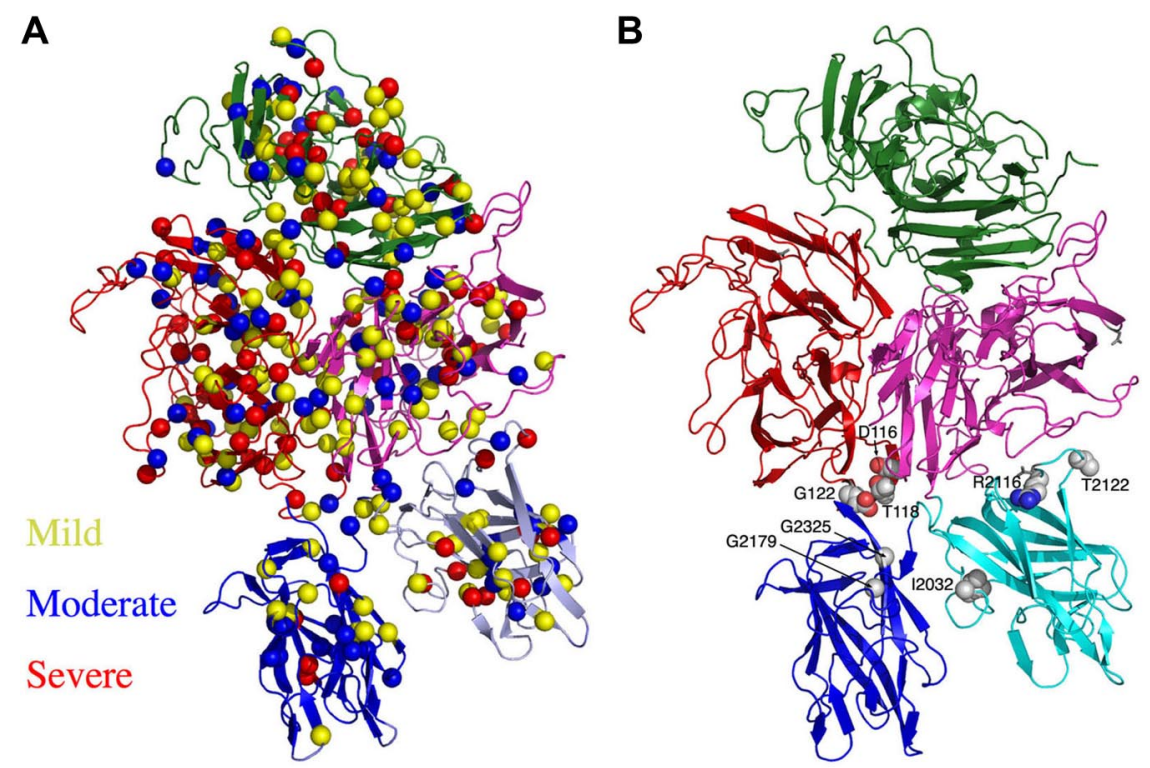

specific chaperones that are highly specific to individual protein targets and expression pathways.

Of 4 potential sites of N-linked glycosylation in the r-fVIII molecule (N42 and N239 in the A1 domain, N1810 in the A3 domain, and $\mathrm{N} 2118$ in the $\mathrm{C} 1$ domain), density is clearly visible for an oligosaccharide structure consistent with a canonical N-linked modification at N239, N1810, and N2118. One of these 3 positions (N1810) is also observed to be glycosylated in the structure of $\mathrm{fV}^{31}$ Of the glycosylation modifications in fVIII, unbiased difference density is particularly striking for extensive well-ordered oligosaccharide structures at both N239 (Figure 2D) and N2118. In both cases, the sugar moieties are located in a domain interface (between $\mathrm{A} 1$ and $\mathrm{A} 2$ for $\mathrm{N} 239$, and between $\mathrm{A} 3$ and $\mathrm{C} 1$ for N2118) and appear to participate in packing and stabilization. In contrast, the oligosaccharide group at N1810 is located near a surface of the A3 domain that is believed to be involved in binding both VWF (through interactions with its $\mathrm{N}$-terminal acidic region) and with LDL receptors involved in fVIII clearance. ${ }^{45}$ Thus, this latter modification, if physiologically relevant, may be important for stabilization and/or clearance of fVIII in vivo.

\section{Discussion}

\section{Hemophilia A mutations}

A deficiency in fVIII clotting activity leads to a common bleeding disorder, hemophilia A, which affects 1 in 5000 males worldwide. ${ }^{46}$ Hemophilia A is an X-linked disorder of variable severity that is due to mutations in the fVIII gene, which is $187 \mathrm{~kb}$ long and contains 26 exons. The genetic lesions resulting in hemophilia A include deletions, exon inversions and translocations, nonsense frame shifts, premature stops, and a large number of missense point mutations, all of which can cause defects in the expression, secretion, and/or half-life of fVIII in circulation. ${ }^{47}$ Alternatively, some hemophilic mutations can generate stable but dysfunctional fVIII. An international database of point mutations that are associated with hemophilia A lists several hundred unique missense mutations within fVIII that all have been observed in vivo and are associated with variable severity of disease symptoms. ${ }^{48}$ These mutations are distributed uniformly across the entire peptide chain of fVIII, regardless of either disease severity or structural domain of the protein (Figure 5A).

A variety of studies have mapped hemophilia A mutations to positions across available models of the fVIII structure. For example, upon determination of the crystal structure of the $\mathrm{C} 2$ domain and generation of a related homology model of the $\mathrm{C} 1$ domain, 57 separate mutations that occur within those regions were analyzed with respect to the correlation between disease severity, effect on circulating levels of r-fVIII, and the position of each residue in the protein fold. ${ }^{29}$ As a general rule, those residues found in core regions of these folded domains, or among surface epitopes known to be critical for procoagulant-binding activities and functions, were more likely to yield dysfunctional and/or destabilized protein. The structure of the full-length r-fVIII protein provides additional detail for these analyses, particularly for mutations located within interfaces between the $\mathrm{C}$ domains and their nearest neighbors in the full-length molecule. For example, 9 separate mutations that yield severe disease symptoms and phenotypes (defined as those that produce less than $1 \%$ of normal circulating fVIII activity) are found on the surface of the A1, C1, and $\mathrm{C} 2$ domains at positions not known to be involved in binding interactions with membranes, VWF, or fIXa. These residues are found to be located, respectively, in the C1-A3 domain interface (R2116 and T2122), in the A1-C2 interface (T118, E122, and D116) and in the C1-C2 interface (G2026, G2179, G2325, and I2032; Figure 5B). These latter residues are of particular interest, as glycine residues are often of great structural importance for formation of functional protein cores and interfaces due to their ability to assume backbone conformations that are otherwise sterically inaccessible.

\section{Engineered fVIII constructs}

Hemophilia A is treated by replacement therapy with concentrated fVIII using typical dosage regiments of 20 to $40 \mathrm{IU} / \mathrm{kg} 3$ times per week, plus prophylactic administration during adverse bleeding episodes. ${ }^{49}$ A major issue that greatly affects replacement therapy efficacy and cost is the instability and rapid clearance of fVIII. Upon activation, the resulting activated fVIIIa is subject to spontaneous decay of its procoagulant activity, attributable to first-order dissociation of its free A2 subunit. ${ }^{50}$ In addition, the 
fVIIIa molecule is cleared by receptor-mediated catabolism, which is mediated by interactions between well-mapped epitopes on the surface of fVIII and 2 receptors from the low-density lipoprotein family (LRP and LDLR; reviewed in Saenko and Pipe ${ }^{49}$ ). Many investigators have hypothesized that improvement of fVIII stability may allow correction of hemostasis in vivo at lower levels of protein, allowing for longer intervals between therapeutic infusions.

A variety of strategies have been tested for stabilization of fVIII and improvement of its circulating lifetime in replacement therapy. ${ }^{49}$ Specifically, fVIII has been altered by chemical modification (primarily via "PEGylated" protein formulations), by elimination of the $\mathrm{B}$ domain and additional protein modifications that covalently trap the A2 domain (either by elimination of a thrombinprocessing site in the fVIII backbone or by introduction of disulfide bonds between A 2 and A 3 domains), and finally by mutagenesis of proposed receptor-binding residues. The structure of r-fVIII provides insight into the design of engineered fVIII variants, particularly with respect to disulfide cross-linked constructs (Figure 4B). Two such constructs have previously been described in some detail: the first containing a pair of cysteines linking residue 664 of the A2 domain to residue 1826 of the A3 domain (Y664C/T1826C), and the second, located in the same region, linking residues 662 and 1828 (M662C/D1828C). ${ }^{51}$ In the crystal structure, these residues pairs are positioned within 2 adjacent loops and display appropriate distances for S-S formation (distance between C-alphas is approximately $7 \AA$ and $13 \AA$, respectively). The use of structural information may be of utility for the creation of new generations of improved fVIII constructs, including those that are stabilized through resculpting the A2 interface solely through increased complementarity of noncovalent contacts and packing using structure-based computational protein design methods.

\section{Antibody inhibitor epitopes}

A significant complication of fVIII replacement therapy is the development of antibody inhibitors. ${ }^{52}$ The most frequent inhibitor incidence occurs on epitopes within the A2 and C2 domains. Inhibitors to the A3 domain and the acidic region between A1 and A2 have also been observed. fVIII antibody inhibitors can block fVIII function in several ways: (1) by blocking the ability of fVIIIa to bind and activate fIXa and fX; (2) by inhibiting the binding of fVIII to VWF and/or negatively charged phospholipid surfaces; or (3) by hindering the activation of fVIII by thrombin (and/or fXa) or the subsequent release of fVIII from VWF.

The antigenic hotspots identified across the surface of r-fVIII generally correspond to the most mobile regions of the r-fVIII structure (Figure S4). A total of 2 epitopes (residues 351-365 in the A1 domain and residues 1674-1684 in the A3 domain) are disordered in the crystal, while another 2 (residues 484-508 in the A2 domain and residues 1814-1819 in the A3 domain) correspond to some of the most elevated B-factors in the structure. In contrast, the $\mathrm{C} 2$ domain, of which at least half has been implicated in inhibitor antibody binding, is internally well ordered; however, the domain as a whole is extensively solvent-exposed and only loosely docked to the remainder of the r-fVIII molecule. The importance of this domain in several critical procoagulant functions may make the existence of corresponding inhibitors particularly significant clinically, facilitating their identification and characterization.

\section{Acknowledgments}

Data were collected at the ALS beamline 5.0.2 with assistance from beamline staff. We thank members of the Strong and Ferre D'Amare laboratories at the Hutchinson Center for invaluable assistance and advice.

Data deposition: The coordinates and structure factor amplitudes for the fVIII structure have been deposited in the Research Collaboratory for Structural Bioinformatics (RCSB) $)^{53,54}$ for immediate release (PDB ID code 2R7E).

This project was funded by National Institutes of Health (NIH) grant R01 HL62570 to B.L.S. P.C.S. was funded by NIH training grant T32 GM08268.

\section{Authorship}

Contribution: B.W.S. and P.C.S. accomplished X-ray data collection and crystallographic modeling; Y.-H.K., C.-H.C., J.-W.H., J.-S.L., and J.K. generated purified r-fVIII; B.L.S., B.W.S., and P.C.S. analyzed the structure and prepared the manuscript; and all authors provided final review of the manuscript and many revisions.

Conflict-of-interest disclosure: C.-H.C., J.-W.H., J.-S.L., and J.K. are employed by the Green Cross Corporation; the fVIII construct described in this manuscript is a commercial product of that company. The remaining authors declare no competing financial interests.

Correspondence: Barry L. Stoddard, Program in Molecular Biophysics, Structure and Design, Division of Basic Sciences, Fred Hutchinson Cancer Research Center, 1100 Fairview Ave N, A3023, Seattle, WA; e-mail: bstoddar@fhcrc.org.

\section{References}

1. Davie EW, Fujikawa K, Kisiel W. The coagulation cascade: initiation, maintenance, and regulation. Biochemistry. 1991;30:10363-10370.

2. Davie EW, Ratnoff OD. Waterfall sequence for intrinsic blood clotting. Science. 1964;145:13101312.

3. Macfarlane RG. An enzyme cascade in the blood clotting mechanism, and its function as a biochemical amplifier. Nature. 1964;202:498-499.

4. Mann KG. Biochemistry and physiology of blood coagulation. Thromb Haemost. 1999;82:165-174.

5. Osterud B, Rapaport SI. Activation of factor IX by the reaction product of tissue factor and factor VII: additional pathway for initiating blood coagulation. Proc Natl Acad Sci U S A. 1977;74:52605264.

6. Walsh PN, Biggs R. The role of platelets in intrin- sic factor-Xa formation. Br J Haematol. 1972;22: 743-760.

7. Davie EW. Biochemical and molecular aspects of the coagulation cascade. Thromb Haemost. 1995;74:1-6.

8. van Dieijen G, Tans G, Rosing J, Hemker HC. The role of phospholipid and factor VIIla in the activation of bovine factor X. J Biol Chem. 1981; 256:3433-3442.

9. Kane WH, Davie EW. Blood coagulation factors and VIII: structural and functional similarities and their relationship to hemorrhagic and thrombotic disorders. Blood. 1988;71:539-555.

10. Lenting PJ, van Mourik JA, Mertens K. The life cycle of coagulation factor VIII in view of its structure and function. Blood. 1998;92:3983-3996.

11. Toole JJ, Knopf JL, Wozney JM, et al. Molecular cloning of a cDNA encoding human antihaemophilic factor. Nature. 1984;312:342-347.

12. Vehar GA, Keyt B, Eaton D, et al. Structure of human factor VIII. Nature. 1984;312:337-342.

13. Pemberton S, Lindley P, Zaitsev V, Card G, Tuddenham EG, Kemball-Cook G. A molecular model for the triplicated A domains of human factor VIII based on the crystal structure of human ceruloplasmin. Blood. 1997;89:2413-2421.

14. Baumgartner $\mathrm{S}$, Hofmann $\mathrm{K}$, Chiquet-Ehrismann $\mathrm{R}$, Bucher $\mathrm{P}$. The discoidin domain family revisited: new members from prokaryotes and a homology-based fold prediction. Protein Sci. 1998; 7:1626-1631.

15. Pellequer JL, Gale AJ, Griffin JH, Getzoff ED. Homology models of the $C$ domains of blood coagulation factors $\mathrm{V}$ and VIII: a proposed membrane 
binding mode for FV and FVIII C2 domains. Blood Cells Mol Dis. 1998;24:448-461.

16. Pratt KP, Shen BW, Takeshima K, Davie EW, Fujikawa K, Stoddard BL. Structure of the C2 domain of human factor VIII at 1.5 A resolution. Nature. 1999;402:439-442.

17. Fuentes-Prior P, Fujikawa K, Pratt KP. New insights into binding interfaces of coagulation factors $\mathrm{V}$ and VIII and their homologues lessons from high resolution crystal structures. Curr Protein Pept Sci. 2002;3:313-339.

18. Eaton $\mathrm{D}$, Rodriguez $\mathrm{H}$, Vehar GA. Proteolytic processing of human factor VIII. Correlation of specific cleavages by thrombin, factor $\mathrm{Xa}$, and activated protein $C$ with activation and inactivation of factor VIII coagulant activity. Biochemistry. 1986; 25:505-512.

19. Foster PA, Fulcher CA, Marti T, Titani K, Zimmerman TS. A major factor VIII binding domain resides within the amino-terminal 272 amino acid residues of von Willebrand factor. J Biol Chem. 1987;262:8443-8446.

20. Lubin IM, Healey JF, Barrow RT, Scandella D, Lollar P. Analysis of the human factor VIII A2 inhibitor epitope by alanine scanning mutagenesis. J Biol Chem. 1997:272:30191-30195.

21. Nogami K, Shima M, Hosokawa K, et al. Role of factor VIII C2 domain in factor VIII binding to factor Xa. J Biol Chem. 1999;274:31000-31007.

22. Saenko E, Sarafanov A, Ananyeva N, et al. Comparison of the properties of phospholipid surfaces formed on HPA and L1 biosensor chips for the binding of the coagulation factor VIII. J Chromatogr A. 2001;921:49-56.

23. Saenko EL, Shima M, Rajalakshmi KJ, Scandella D. A role for the $\mathrm{C} 2$ domain of factor VIII in binding to von Willebrand factor. J Biol Chem. 1994; 269:11601-11605

24. Healey JF, Lubin IM, Nakai $\mathrm{H}$, et al. Residues 484-508 contain a major determinant of the inhibitory epitope in the $\mathrm{A} 2$ domain of human factor VIII. J Biol Chem. 1995;270:14505-14509

25. Scandella D, Gilbert GE, Shima M, et al. Some factor VIII inhibitor antibodies recognize a common epitope corresponding to $\mathrm{C} 2$ domain amino acids 2248 through 2312, which overlap a phospholipid-binding site. Blood. 1995;86:1811-1819.

26. Saenko EL, Scandella D. The acidic region of the factor VIII light chain and the $\mathrm{C} 2$ domain together form the high affinity binding site for von willebrand factor. J Biol Chem. 1997;272:1800718014.

27. Spiegel PC Jr, Jacquemin M, Saint-Remy JM, Stoddard BL, Pratt KP. Structure of a factor VIII C2 domain-immunoglobulin G4kappa Fab com- plex: identification of an inhibitory antibody epitope on the surface of factor VIII. Blood. 2001; 98:13-19.

28. Macedo-Ribeiro S, Bode W, Huber R, et al. Crystal structures of the membrane-binding $\mathrm{C} 2$ domain of human coagulation factor $\mathrm{V}$. Nature. 1999;402:434-439.

29. Liu ML, Shen BW, Nakaya S, et al. Hemophilic factor VIII C1- and C2-domain missense mutations and their modeling to the 1.5-angstrom human C2-domain crystal structure. Blood. 2000; 96:979-987.

30. Stoilova-McPhie S, Villoutreix BO, Mertens K, Kemball-Cook G, Holzenburg A. 3-Dimensional structure of membrane-bound coagulation factor VIII: modeling of the factor VIII heterodimer within a 3-dimensional density map derived by electron crystallography. Blood. 2002;99:1215-1223.

31. Adams TE, Hockin MF, Mann KG, Everse SJ. The crystal structure of activated protein C-inactivated bovine factor Va: Implications for cofactor function. Proc Natl Acad Sci U S A. 2004;101:89188923.

32. Oh HK, Lee JM, Byun TH, Park SY, Kim YH. Purification of recombinant human B-domain-deleted factor VIII using anti-factor VIII monoclonal antibody selected by the surface plasmon resonance biosensor. Biotechnol Prog. 2001;17:1119-1127.

33. Pflugrath JW. The finer things in X-ray diffraction data collection. Acta Crystallogr D Biol Crystallogr. 1999;55:1718-1725.

34. CCP4: The SERC (UK) collaborative computing project No. 4, a suite of programs for protein crystallography. Warrington, United Kingdom: Daresbury Laboratory; 1979.

35. Kissinger CR, Gehlhaar DK, Fogel DB. Rapid automated molecular replacement by evolutionary search. Acta Crystallogr D Biol Crystallogr. 1999; 55:484-491.

36. Bento I, Peixoto C, Zaitsev VN, Lindley PF. Ceruloplasmin revisited: structural and functional roles of various metal cation binding sites. Acta Crystallogr D Biol Crystallogr. 2007;63:240-247

37. Kim DE, Chivian D, Baker D. Protein structure prediction and analysis using the Robetta server. Nucleic Acids Res. 2004;32:W526-531.

38. Brunger AT, Adams PD, Clore GM, et al. Crystallography \& NMR system: a new software suite for macromolecular structure determination. Acta Crystallogr D Biol Crystallogr. 1998;54:905-921.

39. Emsley P, Cowtan K. Coot: model-building tools for molecular graphics. Acta Crystallogr D Biol Crystallogr. 2004;60:2126-2132.

40. Spiegel PC, Jr., Murphy P, Stoddard BL. Surfaceexposed hemophilic mutations across the factor
VIII C2 domain have variable effects on stability and binding activities. J Biol Chem. 2004;279: 53691-53698.

41. Takeshima K, Smith C, Tait J, Fujikawa K. The preparation and phospholipid binding property of the C2 domain of human factor VIII. Thromb Haemost. 2003;89:788-794.

42. Tagliavacca L, Moon N, Dunham WR, Kaufman RJ. Identification and functional requirement of $\mathrm{Cu}(\mathrm{I})$ and its ligands within coagulation factor VIII. J Biol Chem. 1997;272:27428-27434.

43. Mann KG, Lawler CM, Vehar GA, Church WR. Coagulation factor $\mathrm{V}$ contains copper ion. J Biol Chem. 1984;259:12949-12951.

44. Wakabayashi H, Zhou Z, Nogami K, et al. pHdependent association of factor VIII chains: enhancement of affinity at physiological $\mathrm{pH}$ by Cu2 + . Biochim Biophys Acta. 2006;1764:10941101.

45. Bovenschen N, Boertjes RC, van Stempvoort G, et al. Low density lipoprotein receptor-related protein and factor IXa share structural requirements for binding to the $\mathrm{A} 3$ domain of coagulation factor VIII. J Biol Chem. 2003;278:9370-9377.

46. Rosendaal FR, Smit C, Briet E. Hemophilia treatment in historical perspective: a review of medical and social developments. Ann Hematol. 1990;62: 5-15.

47. Hoyer LW. Hemophilia A. N Engl J Med. 1994; 330:38-47.

48. Tuddenham EG, Schwaab R, Seehafer J, et al. Haemophilia A: database of nucleotide substitutions, deletions, insertions and rearrangements of the factor VIII gene, second edition. Nucleic Acids Res. 1994;22:4851-4868.

49. Saenko E, Pipe SW. Strategies towards a longer acting factor VIII. Haemophilia. 2006;12:42-51.

50. Fay PJ, Smudzin TM. Characterization of theinteraction between the $\mathrm{A} 2$ subunit and $\mathrm{A} 1 / \mathrm{A} 3-$ C1-C2 dimer in human factor VIIla. J Biol Chem 1992;267:13246-13250.

51. Gale AJ, Xu X, Pellequer JL, Getzoff ED, Griffin $\mathrm{JH}$. Interdomain engineered disulfide bond permitting elucidation of mechanisms of inactivation of coagulation factor Va by activated protein $\mathrm{C}$. Protein Sci. 2002;11:2091-2101.

52. Spiegel PC Jr, Stoddard BL. Optimization of factor VIII replacement therapy: can structural studies help in evading antibody inhibitors? $\mathrm{Br} \mathrm{J}$ Haematol. 2002;119:310-322.

53. Berman HM, Westbrook J, Feng Z, et al. The Protein Data Bank. Nucleic Acids Res. 2000;28:235242.

54. Research Collaboratory for Structural Bioformatics. Protein Data Bank. http://www.rcsb.org/pdb/ home/home.do, accessed September 28, 2007 


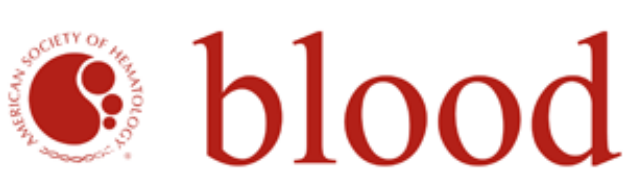

2008 111: 1240-1247

doi:10.1182/blood-2007-08-109918 originally published online October 26, 2007

\section{The tertiary structure and domain organization of coagulation factor VIII}

Betty W. Shen, Paul Clint Spiegel, Chong-Hwan Chang, Jae-Wook Huh, Jung-Sik Lee, Jeanman Kim, Young-Ho Kim and Barry L. Stoddard

Updated information and services can be found at:

http://www.bloodjournal.org/content/111/3/1240.full.html

Articles on similar topics can be found in the following Blood collections

Hemostasis, Thrombosis, and Vascular Biology (2494 articles)

Information about reproducing this article in parts or in its entirety may be found online at:

http://www.bloodjournal.org/site/misc/rights.xhtml\#repub_requests

Information about ordering reprints may be found online at:

http://www.bloodjournal.org/site/misc/rights.xhtml\#reprints

Information about subscriptions and ASH membership may be found online at:

http://www.bloodjournal.org/site/subscriptions/index.xhtml

Blood (print ISSN 0006-4971, online ISSN 1528-0020), is published weekly by the American Society of Hematology, 2021 L St, NW, Suite 900, Washington DC 20036.

Copyright 2011 by The American Society of Hematology; all rights reserved. 